\title{
Structural, intramolecular hydrogen bonding and vibrational studies on 3-amino-4-methoxy benzamide using density functional theory
}

\author{
G SUBHAPRIYA $^{\mathrm{a}}$, S KALYANARAMAN ${ }^{\mathrm{a}, *}, \mathrm{~S} \mathrm{GANDHIMATHI}^{\mathrm{b}}, \mathrm{N}^{\mathrm{S}}$ SURUMBARKUZHALI $^{\mathrm{c}}$ \\ and V KRISHNAKUMAR ${ }^{\mathrm{d}}$ \\ ${ }^{a}$ PG \& Research Department of Physics, Sri Paramakalyani College, Alwarkurichi 627 412, India \\ ${ }^{b}$ Department of Chemistry, Sri Paramakalyani College, Alwarkurichi 627412, India \\ ${ }^{c}$ Department of Physics, Government Arts College (Autonomous), Salem 636 007, India \\ ${ }^{\mathrm{d}}$ Department of Physics, Periyar University, Salem 636 011, India \\ Email: mayura_priya2003@yahoo.co.in
}

MS received 3 May 2016; accepted 5 January 2017

\begin{abstract}
An extensive theoretical study on the molecular structure and vibrational analysis of 3-amino-4methoxy benzamide (3A4MBA) was undertaken using density functional theoretical (DFT) method. The possibility of formation of intramolecular hydrogen bonding was identified from structural parameter analysis and confirmed with the natural bond orbital (NBO), molecular electrostatic potential (MEP) and natural charge analysis. The harmonic oscillator model of aromaticity (HOMA) index elucidated the impact of hydrogen bonding in the ring. Intramolecular hydrogen bonding energy has been calculated from topological study. The low wavenumber vibrational modes obtained from experimental FT-Raman spectrum also supported the presence of hydrogen bonding. Bands are assigned for vibrational frequencies using DFT/B3LYP/6-311++G** level of theory.
\end{abstract}

Keywords. Amino methoxy benzamide; DFT; vibrational analysis; intramolecular hydrogen bonding.

\section{Introduction}

Amides contain the $-\mathrm{CONH}_{2}$ group in which a carbon atom is attached to oxygen by a covalent double bond. ${ }^{1}$ Benzamide is the simplest aromatic carboxylic amide, which is used in the synthesis of various organic compounds. Benzamide is slightly soluble in water but easily soluble in many organic solvents. ${ }^{2}$

Benzamide derivatives have therapeutic utility. ${ }^{3}$ Benzamide derivatives are known to cure antipsychotic and antiemetic ailments. Owing to its neurotropic characteristics, this class of compounds finds use in imaging melanoma and melanoma meta states. ${ }^{4} \mathrm{~N}, \mathrm{~N}$-diethyl benzamide and $\mathrm{N}, \mathrm{N}$-diethyl 3-methyl benzamide are used in mosquito repellents. A benzamide derivative is used as an antipsychotic drug for many depressive disorders. ${ }^{5}$ Owing to various applications of benzamide and its derivatives, ${ }^{5}$ identifying the structure and vibrations of benzamide molecules by quantum chemical calculations are necessary for understanding many of its related properties. ${ }^{6-12}$ The present work deals with 3-amino-4-methoxy benzamide (3A4MBA). This is an intermediate product of dyes and pigments. Structural studies on this molecule are scanty. Hence, an extended

\footnotetext{
*For correspondence
}

theoretical analysis on the structural and vibrational properties of the 3A4MBA molecule is undertaken using density functional theory (DFT) calculations. An effort has been made to calculate the $\mathrm{N}-\mathrm{H}-$ - - $^{-}$ intramolecular hydrogen bonding, its nature, bond distances and related frequencies by calculating the topological parameters at the bond critical points using atoms-in-molecule theory.

\section{Experimental and Computational}

The fine polycrystalline sample of 3A4MBA was procured from Alfa Aesar Company with a stated purity of $98 \%$ and used as such. Infrared spectrum of the compound by $\mathrm{KBr}$ pellet method was recorded with JASCO FT-IR 4100 spectrometer in the region $400-4000 \mathrm{~cm}^{-1}$. The FT-Raman spectrum of 3A4MBA was recorded on a BRUKER IFS-66V model interferometer equipped with FRA-106 FT-Raman equipment. The spectrum was recorded in the range $4000-0 \mathrm{~cm}^{-1}$. For Stokes region, $1064 \mathrm{~nm}$ line of a Nd:YAG laser was used for the excitation with an operating power of $200 \mathrm{~mW}$. The reported transitions are accurate in the order $\pm 1 \mathrm{~cm}^{-1}$.

The molecular geometries of 3A4MBA were fully optimized with DFT methods using the $6-311++\mathrm{G}^{* *}$ basis set and further calculations were carried out with the same basis set. Theoretical calculations were performed with Gaussian- $03^{13}$ program package, invoking gradient geometry 
optimization on Intel core $15 / 2.50 \mathrm{GHz}$ processor. DFT with the three-parameter hybrid function (B3) for the exchange part and the Lee-Yang-Parr (LYP) correlation function ${ }^{14-16}$ were utilised for three-dimensional potential energy scan (3DPES), molecular structure optimization, vibrational frequencies, natural bond orbital (NBO), molecular electrostatic potential (MEP) and UV-Vis spectral analysis.

\section{Results and Discussion}

\subsection{Conformational study}

Estimation of hydrogen bonding in the present compound is due to the presence of adjacent amino group and methoxy group in the aromatic ring. To find out the impact of hydrogen bonding in defining conformational flexibility of the molecule along with its type of bonding, HF/6-31G** calculations were carried out by

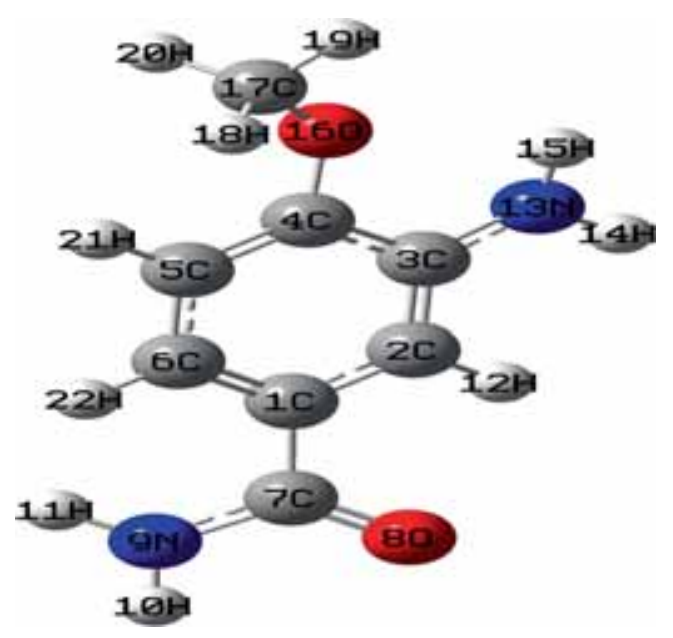

Figure 1. Structure of 3A4MBA. applying rotations in the amino and methoxy groups. Minimum energy corresponding to a particular geometry was obtained using a three dimensional potential energy scan. The structure of 3A4MBA molecule is shown in Figure 1. The dihedral angle of H18-C17O16-C4 of methoxy group and H15-N13-C3-C4 of amino group with the aromatic ring was varied by every $20^{\circ}$. Two 3DPES were performed, one for the amide group $\mathrm{C} 2-\mathrm{C} 1-\mathrm{C} 7-\mathrm{O} 8$ with the dihedral angle of $180^{\circ}$ (Figure 2) and other one for C2-C1-C7-O8 with the dihedral angle of $0^{\circ}$ (Figure 3 ). The total energies obtained for these possible conformers are listed in Table 1. From the two 3DPES figures, we observed more number of saddle points, and 11 optimized minimum energy values for each scan and are been tabulated. Minimum energy of -567.1727 Hartrees was obtained for the conformer number 332 when C2-C1C7-O8 has the dihedral angle of $180^{\circ}$ and the methoxy group H18-C17-O16-C4 at $180^{\circ}$ and the amino group $\mathrm{H} 15-\mathrm{N} 13-\mathrm{C} 3-\mathrm{C} 4$ at $360^{\circ}$. There is no imaginary frequencies in the optimized structure $\left(\mathrm{N}_{\mathrm{im}}=0\right)$ and positive harmonic vibrations are only obtained for the minimum energy conformer number 332, indicating that conformer number 332 is the most stable structure.

\subsection{Structural properties}

The structure and numbering scheme for atoms of 3A4MBA are shown in Figure 4. The optimized structural parameters for the geometry of 3A4MBA determined at B3LYP/6-311++G** level are presented in Table S1 (in Supplementary Information) for the molecule shown in Figure 4. Adjacent methoxy and amino groups indicate the possibility of formation of intramolecular hydrogen bonding.
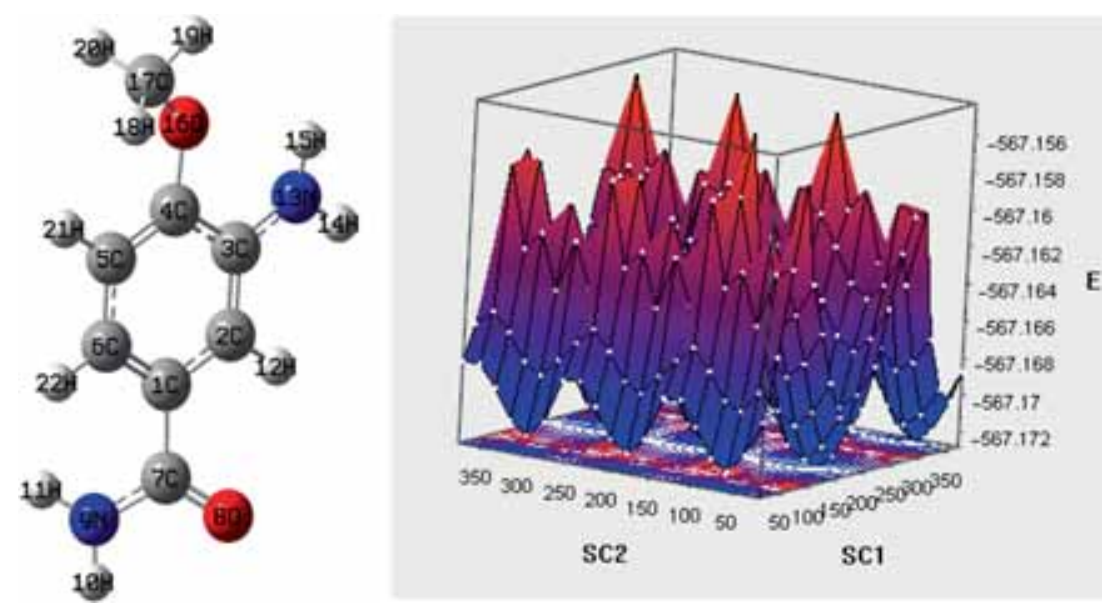

Figure 2. Three dimensional potential energy scan for $\mathrm{C} 2-\mathrm{C} 1-\mathrm{C} 7-\mathrm{O} 8$ dihedral angle $180^{\circ}$. Here, SC1 is the dihedral angle of C4-O16-C17-H18, SC2 is the dihedral angle of C4-C3-N13-H15. 


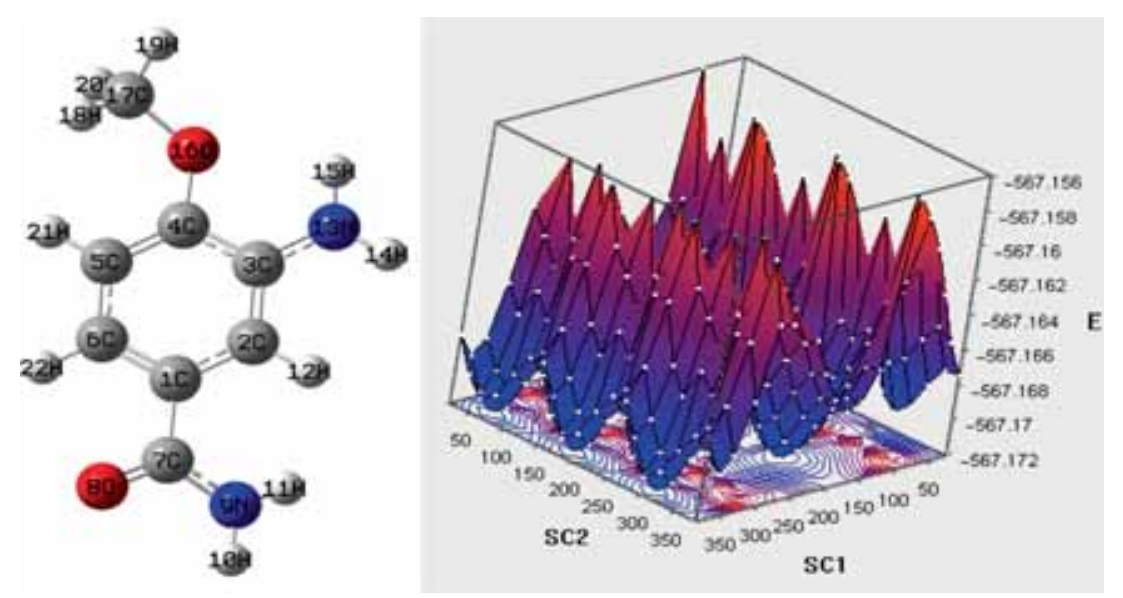

Figure 3. Three dimensional potential energy scan for $\mathrm{C} 2-\mathrm{C} 1-\mathrm{C} 7-\mathrm{O} 8$ dihedral angle $0^{\circ}$. Here, $\mathrm{SC} 1$ is the dihedral angle of C4-O16-C17-H18, SC2 is the dihedral angle of C4-C3-N13-H15.

Table 1. Geometrical parameters of 3-amino-4-methoxy benzamide and energy.

\begin{tabular}{|c|c|c|c|c|}
\hline Conformer & $\begin{array}{l}\mathrm{SC} 1 \text { in } \\
\text { degree }\end{array}$ & $\begin{array}{l}\mathrm{SC} 2 \text { in } \\
\text { degree }\end{array}$ & $\begin{array}{l}\text { Total energies } \\
\mathrm{kJ} / \mathrm{mol}\end{array}$ & $\begin{array}{c}\text { Energy difference } \\
\Delta \mathrm{E} \text { With respect to conformer } 33\end{array}$ \\
\hline \multicolumn{5}{|c|}{$\mathrm{C} 2-\mathrm{C} 1-\mathrm{C} 7-\mathrm{O} 8$ has the dihedral angle of $180^{\circ}$} \\
\hline 332 & 180 & 360 & -1489112.0403 & 0.0000 \\
\hline 326 & 60 & 360 & -1489112.0336 & -0.0067 \\
\hline 338 & 300 & 360 & -1489112.0323 & -0.0080 \\
\hline 161 & 180 & 180 & -1489112.0286 & -0.0117 \\
\hline 155 & 60 & 180 & -1489112.0217 & -0.0186 \\
\hline 167 & 300 & 180 & -1489112.0209 & -0.0195 \\
\hline 142 & 180 & 160 & -1489110.4296 & -1.6107 \\
\hline 148 & 300 & 160 & -1489110.4269 & -1.6135 \\
\hline 136 & 60 & 160 & -1489110.4176 & -1.6228 \\
\hline 166 & 280 & 180 & -1489110.2565 & -1.7838 \\
\hline 337 & 280 & 360 & -1489110.2561 & -1.7843 \\
\hline \multicolumn{5}{|c|}{$\mathrm{C} 2-\mathrm{C} 1-\mathrm{C} 7-\mathrm{O} 8$ has the dihedral angle of $0^{\circ}$} \\
\hline 332 & 180 & 360 & -1489110.3797 & -1.6607 \\
\hline 326 & 60 & 360 & -1489110.3776 & -1.6628 \\
\hline 338 & 300 & 360 & -1489110.3671 & -1.6733 \\
\hline 161 & 180 & 180 & -1489110.3669 & -1.6734 \\
\hline 155 & 60 & 180 & -1489110.3646 & -1.6758 \\
\hline 167 & 300 & 180 & -1489110.3549 & -1.6855 \\
\hline 142 & 180 & 160 & -1489109.1714 & -2.8689 \\
\hline 148 & 300 & 160 & -1489109.1646 & -2.8758 \\
\hline 136 & 60 & 160 & -1489109.1638 & -2.8765 \\
\hline 337 & 280 & 360 & -1489108.6368 & -3.4036 \\
\hline 166 & 280 & 180 & -1489108.6325 & -3.4078 \\
\hline
\end{tabular}

From the structural data, it was observed that the calculated $\mathrm{C}-\mathrm{C}$ bond distances of the ring carbon atoms and the $\mathrm{C}-\mathrm{H}$ bond lengths were consistent with the literature values except for $\mathrm{C} 3-\mathrm{C} 4$ bond. ${ }^{17}$ The substitution of methoxy and amino groups were responsible for the elongation of $\mathrm{C} 3-\mathrm{C} 4$ bond. $\mathrm{C} 4-\mathrm{O} 16$ bond length also got elongated (1.37 $\AA$ compared to the normal bond length of $1.347 \AA)^{17}$ which might be due to the presence of intramolecular hydrogen bond with the adjacent amino group. The distortion in the symmetry of the ring is caused by intra molecular hydrogen bond. Some of the endocyclic angles of the ring $\mathrm{C} 3-\mathrm{C} 4-\mathrm{C} 5, \mathrm{C} 4-$ $\mathrm{C} 5-\mathrm{C} 6$ and $\mathrm{C} 5-\mathrm{C} 6-\mathrm{C} 1$ were $120^{\circ}$ whereas the angles of $\mathrm{C} 1-\mathrm{C} 2-\mathrm{C} 3, \mathrm{C} 2-\mathrm{C} 3-\mathrm{C} 4$ and $\mathrm{C} 6-\mathrm{C} 1-\mathrm{C} 2$ are $121.5^{\circ}$, $118.3^{\circ}$ and $119.3^{\circ}$, respectively. In addition, the bond length of H15- - -O16 was found to be $2.431 \AA$ which would validate the formation of intramolecular hydrogen bonding. Deviation from computation occurred 


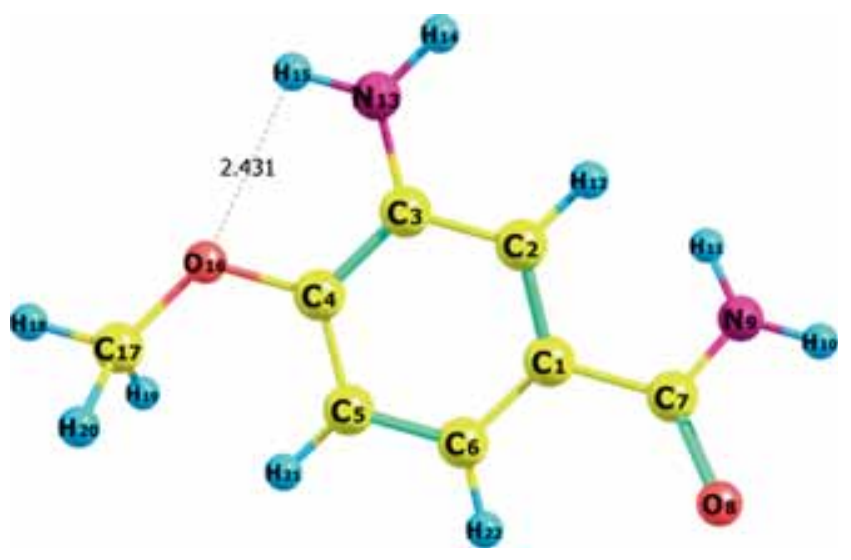

Figure 4. Optimized molecular structure of 3-amino-4methoxy benzamide.

only at the exocyclic angle $\mathrm{C} 2-\mathrm{C} 3-\mathrm{C} 4$, as the molecule in the present study possessed intramolecular hydrogen bonding.

\subsection{Natural bond orbital analysis}

NBO analysis was carried out to explain the charge transfer and delocalization of charges due to inter- and intramolecular interaction among bonds. Delocalization of electron occurred between the occupied Lewis-type and non-Lewis-type NBO orbitals. ${ }^{17,18}$ The delocalization would explain the details of conjugation in the whole system. Transitions for which stabilization energy is high are selected for the molecule and are given in Table 2.

Interestingly, charge transfer was identified through the predicted intramolecular hydrogen bonding O16- - H15-N13 in the geometrical structure of the molecule. The lone pair transition LP $(\mathrm{O} 16) \rightarrow \sigma^{*}(\mathrm{~N} 13-\mathrm{H} 15)$ had very small hyper conjugative interaction energy/ stabilization energy of $1.25 \mathrm{kcal} / \mathrm{mol}$ with the donor occupancy of 1.96108 and acceptor occupancy of 0.01090 which would confirm the weak nature of intramolecular interaction.

\subsection{Molecular electrostatic potential}

The MEP diagram with the contour of the molecule is shown in Figure 5. It clearly depicts the reactive sites of oxygen and hydrogen atoms which emphasize the electrophilic and nucleophilic attacks in the molecule. These sites have been identified with different colours for better clarity. ${ }^{19,20}$ The localization of the negative region over the oxygen atom $(\mathrm{O} 8)$ of the carbonyl moiety and the maximum positive region on the hydrogen atom (H10 and H11) of the amide group clearly indicate a possible site for a nucleophilic attack. The oxygen atom (O16) in the methoxy group lost more of its red colour grading than $\mathrm{O} 8$ atom due to the weak intramolecular hydrogen bonding between $\mathrm{O} 16$ and H15 in the amino group.

\subsection{Natural charges}

Natural atomic charges of the molecule were calculated by DFT method and are presented in S2 (in Supplementary Information). The observed charge value of $\mathrm{O} 8$ was -0.62462 and that of $\mathrm{O} 16$ was -0.53873 . The decreased value of charge in oxygen atom (O16) may be due to the intramolecular hydrogen bond formation. In addition, the carbon atomic charges were found to be positive and negative, while hydrogen atomic charges were only positive. At the same time, the recorded negative charge values of $\mathrm{O}$ and $\mathrm{N}$ atoms indicate a charge transfer from carbon to these atoms.

Table 2. Occupancies of bonding and antibonding orbitals.

\begin{tabular}{lcccccc}
\hline Donor NBO & Type of bond & Occupancy & Acceptor NBO & Type of bond & Occupancy & Energy kcal/mol \\
\hline C1-C6 & $\pi(2)$ & 1.69078 & C2-C3 & $\pi *(2)$ & 0.36318 & 17.9 \\
C1-C6 & $\pi(2)$ & 1.69078 & C4-C5 & $\pi *(2)$ & 0.39209 & 18.760 \\
C1-C6 & $\pi(2)$ & 1.69078 & C7-O8 & $\pi *(2)$ & 0.27498 & 18 \\
C2-C3 & $\pi(2)$ & 1.66068 & C1-C6 & $\pi *(2)$ & 0.39824 & 20.47 \\
C2-C3 & $\pi(2)$ & 1.66068 & C4-C5 & $\pi *(2)$ & 0.39209 & 19.63 \\
C4-C5 & $\pi(2)$ & 1.7028 & C1-C6 & $\pi *(2)$ & 0.39824 & 18.23 \\
C4-C5 & $\pi(2)$ & 1.7028 & C2-C3 & $\pi *(2)$ & 0.36318 & 16.67 \\
O8 & $\mathrm{n}(2)$ & 1.86696 & C1-C7 & $\sigma *(1)$ & 0.06861 & 19.51 \\
O8 & $\mathrm{n}(2)$ & 1.86696 & $\mathrm{C} 7-\mathrm{N} 9$ & $\sigma *(1)$ & 0.06829 & 25.24 \\
N9 & $\mathrm{n}(1)$ & 1.77998 & $\mathrm{C} 7-\mathrm{O} 8$ & $\pi *(2)$ & 0.27498 & 39.95 \\
N13 & $\mathrm{n}(1)$ & 1.83986 & $\mathrm{C} 2-\mathrm{C} 3$ & $\pi *(2)$ & 0.36318 & 27.41 \\
O16 & $\mathrm{n}(2)$ & 1.84802 & $\mathrm{C} 4-\mathrm{C} 5$ & $\pi *(2)$ & 0.39209 \\
O16 & $\mathrm{n}(1)$ & 1.96108 & $\mathrm{~N} 13-\mathrm{H} 15$ & $\sigma *(1)$ & 0.0109 & 23.77 \\
\hline
\end{tabular}



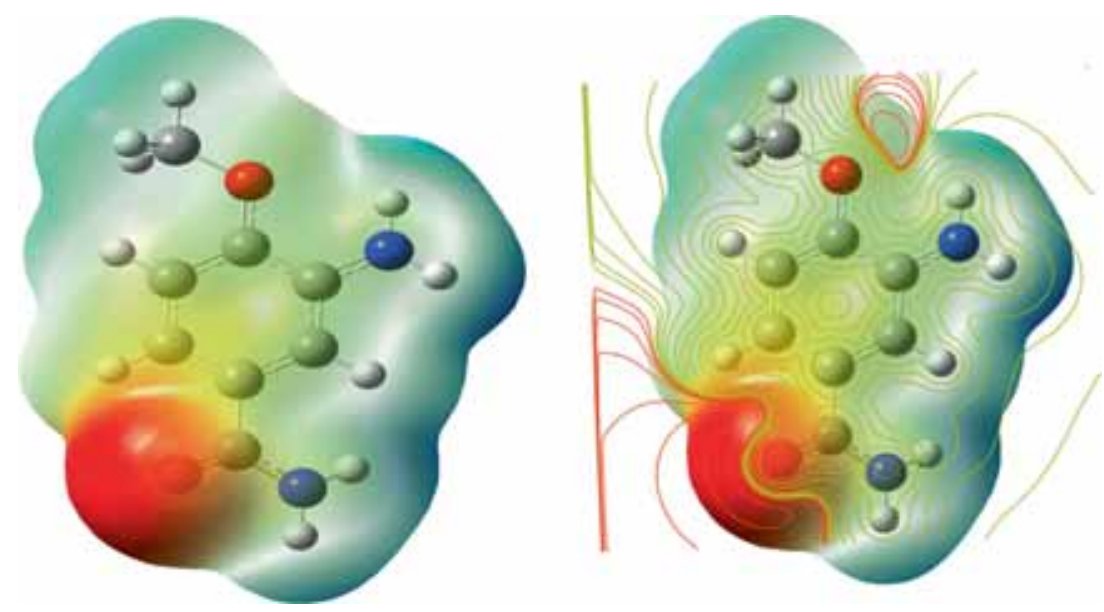

Figure 5. Molecular electrostatic potential and contour of 3-amino-4-methoxy benzamide.
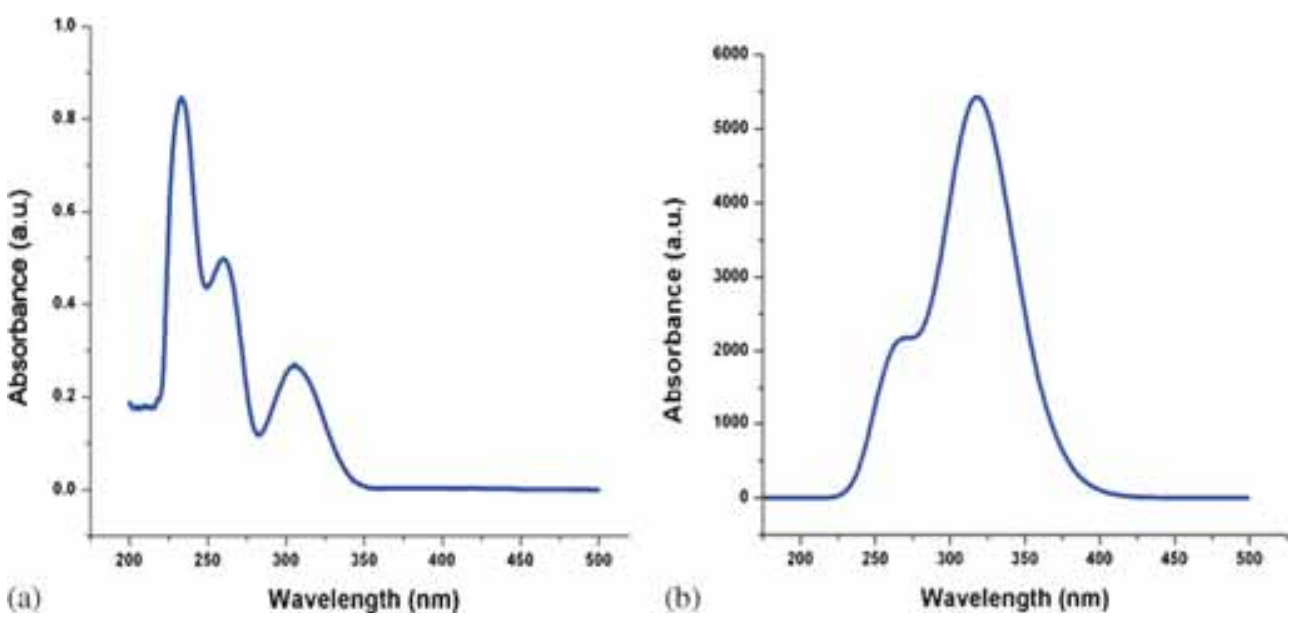

Figure 6. Experimental and calculated UV-Vis spectra of 3-amino-4-methoxy benzamide. (A) Experimental (Ethanol conc. $1 \times 10^{-5} \mathrm{M}$; pathlength, $1 \mathrm{~cm}$ ); (B) Calculated.

\subsection{Aromaticity}

Aromaticity is a measure of the $\pi$-electron delocalization in cyclic systems. Of the many quantitative measures of aromaticity in the ring, harmonic oscillator model of aromaticity (HOMA) can be used. The HOMA index can be analytically separated into two independent terms, namely GEO and EN, describing quantitatively whether the decrease of $\pi$-electron delocalization is due to an increase of bond alternation (GEO) or the elongation of the mean bond length (EN). The HOMA inde ${ }^{21}$ is defined as:

$$
\begin{aligned}
H O M A & =1-\left\lfloor\alpha\left(R_{o p t}-R_{a v}\right)^{2}+\frac{\alpha}{n} \sum\left(R_{a v}-R_{i}\right)^{2}\right\rfloor \\
& =1-E N-G E O
\end{aligned}
$$

Where,

$$
\begin{gathered}
E N=\alpha\left(R_{o p t}-R_{a v}\right)^{2} \\
G E O=\frac{\alpha}{n} \sum\left(R_{a v}-R_{i}\right)^{2}
\end{gathered}
$$

$\mathrm{R}_{\mathrm{av}}$, the average bond length was found to be $1.393 \AA$ for the ring $\mathrm{C}-\mathrm{C}$ while $\mathrm{R}_{\mathrm{i}}$ the running bond length was $1.41 \AA$. The normalisation constant $\alpha$ for $\mathrm{C}-\mathrm{C}$ bonds was 257.7 and $n$ is the number of bonds which is 6 . The calculated GEO was 0.01241 and EN was 0.00644 which gave rise to a HOMA index of 0.98115 for the present system. This only indicated that there was no large deviation from aromaticity in the benzene ring. Hence, the geometry of the molecule was not altered much by intramolecular hydrogen bonding, giving an indication of its weakness in the present system.

\subsection{Electronic structure and UV spectrum}

Using the fully ground state optimized structure, TDDFT calculation was used at B3LYP/6-311++ $\mathrm{G}^{* *}$ level in CPCM model for solvent ethanol to determine the low lying excited state of the molecule. The simulated and experimental UV-Vis spectra, absorbance $v s$ wavelength, are displayed in Figure 6. The possible 
computed transitions are listed in Table 3 . The $\pi \rightarrow \pi^{*}$ transition in the aromatic ring was observed experimentally at $235 \mathrm{~nm}$ and by TD-DFT method, at $264 \mathrm{~nm}$ with an oscillator strength $f=0.0411$. The absorbance at $318 \mathrm{~nm}$ with an oscillator strength $\mathrm{f}=0.1330$ belongs the $\pi \rightarrow \pi^{*}$ transition caused by $\mathrm{C}=\mathrm{O}$ in the amide group which is experimentally found at $305 \mathrm{~nm}$.

\subsection{Vibrational analysis}

MOLVIB version V7.0-G77 was utilized for scaling of the observed frequencies for total energy distribution. The calculated Raman activities $\left(\mathrm{S}_{\mathrm{i}}\right)$ were suitably adjusted by the scaling procedure with MOLVIB and subsequently, the relative Raman intensities $\left(\mathrm{I}_{\mathrm{i}}\right)$ were converted..$^{22-24}$ The full set of 84 standard internal coordinates defined for 3A4MBA are given in Table S3 (in SI), from which constructions of a non-redundant set of 84 local symmetry coordinates were undertaken. ${ }^{25,26}$ These coordinates are summarized in Table S4 (in SI). The title molecule has 22 atoms with 60 fundamental modes of vibrations which belong to $\mathrm{C} 1$ point group symmetry. The FT-IR and FT-Raman spectra are presented in Figures 7 and 8, respectively, which give a visual comparison between the observed and simulated spectra. The experimental assignments of FT-IR and FT-Raman for different vibrational frequencies of 3A4MBA are shown in Table S5 (in SI). The root mean square (RMS) values of frequencies were found ${ }^{23}$ and the error (unscaled /B3LYP/6-311++ $\mathrm{G}^{* *}$ ) obtained for 3A4MBA molecule was found to be $76.7 \mathrm{~cm}^{-1}$. The observed wavenumbers were reproduced using scaling factors and optimized through least square refinement algorithm. The average RMS deviation obtained is $6.4 \mathrm{~cm}^{-1}$.

3.8a Ring vibrations: There are six equivalent $\mathrm{C}-\mathrm{C}$ bonds in benzene and, relevantly, six $\mathrm{C}-\mathrm{C}$ stretching vibrations, in addition to, in-plane and out-ofplane bending $\mathrm{C}-\mathrm{C}-\mathrm{C}$ based bond vibrations in the ring carbons. The skeletal $\mathrm{C}-\mathrm{C}$ stretching bands of benzene derivatives usually occur in the region $1400-1650 \mathrm{~cm}^{-1} \cdot{ }^{27}$ The bands observed at 1631, 1614, $1606,1561,1515,1486 \mathrm{~cm}^{-1}$ were assigned to $\mathrm{C}-\mathrm{C}$

Table 3. Calculated absorption wavelength $(\lambda)$, excitation energies (E), oscillator strength (f) of 3A4MBA by the TD-DFT method.

\begin{tabular}{lccc}
\hline $\begin{array}{l}\text { Experimental } \\
\text { wavelength (nm) }\end{array}$ & $\begin{array}{c}\text { Calculated wavelength } \\
(\mathrm{nm})\end{array}$ & Energy (eV) & Oscillator strength (f) \\
\hline 235 & 264 & 4.68 & 0.0411 \\
305 & 318 & 3.89 & 0.133 \\
\hline
\end{tabular}

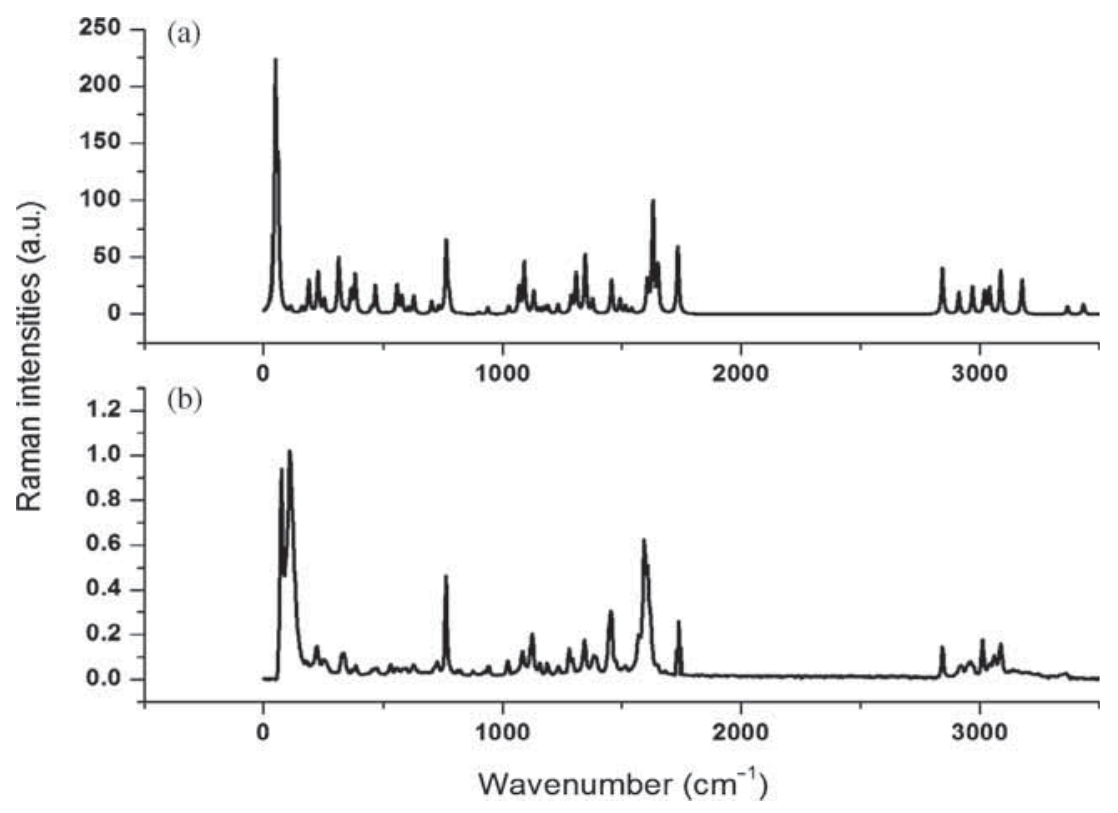

Figure 7. FT-Raman spectra of 3-amino-4-methoxy benzamide. (a) Calculated; (b) Observed. 


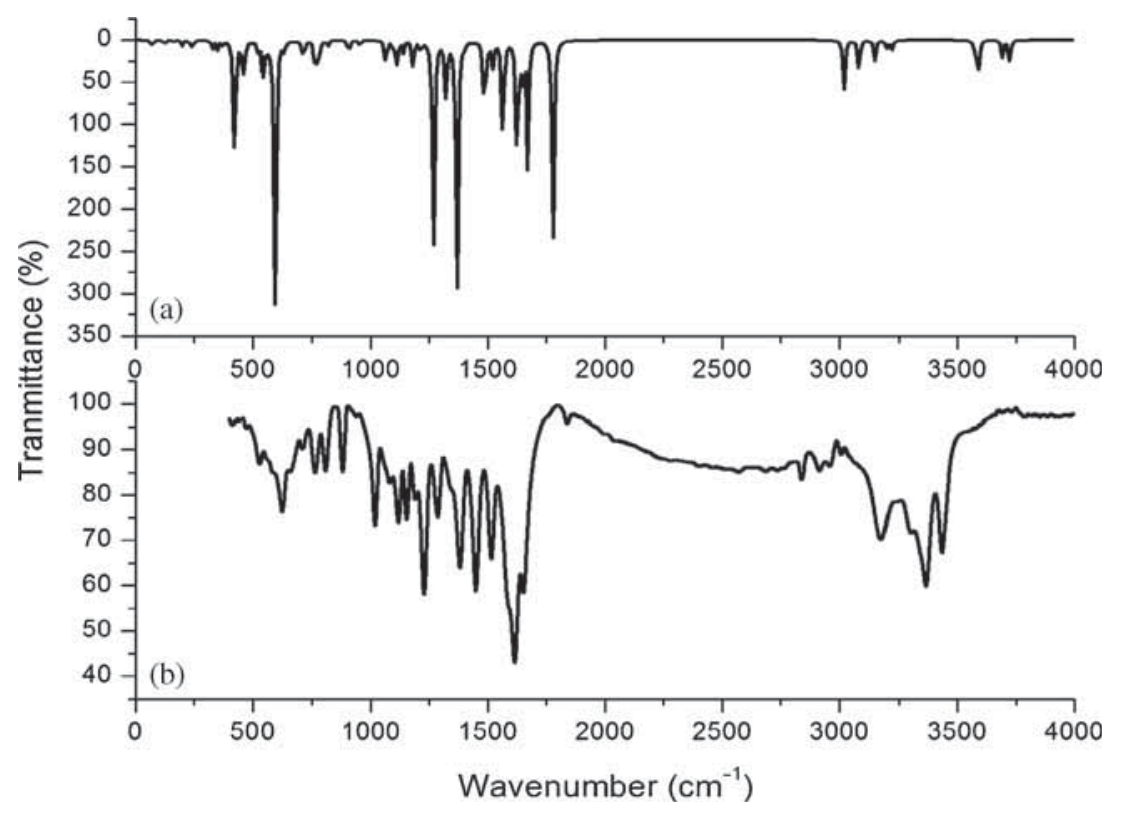

Figure 8. FT-IR spectra of 3-amino-4-methoxy benzamide. (a) Calculated; (b) Observed.

stretching vibration and were in good agreement with the theoretically computed values.

3.8b Amine and Amide group vibrations: In aromatic primary amines, asymmetric and symmetric stretching of vibrations of $\mathrm{N}-\mathrm{H}$ are in the regions $3520-3420 \mathrm{~cm}^{-1}$ and $3420-3340 \mathrm{~cm}^{-1}$, respectively. ${ }^{28}$ Owing to the result of intramolecular hydrogen bonding, the asymmetric stretching of $\mathrm{N}-\mathrm{H}$ vibration got slightly lowered from the higher value to $3366 \mathrm{~cm}^{-1}$ in this molecule. The theoretically scaled down frequency at $3366 \mathrm{~cm}^{-1}$ by B3LYP/6-311++G** method implied a good agreement with the experimentally observed value. In primary amides, two sharp bands observed in the region $3375-3320 \mathrm{~cm}^{-1}$ and $3205-3155 \mathrm{~cm}^{-1}$ were assigned to $\mathrm{N}-\mathrm{H}$ asymmetric and symmetric stretching vibrations, respectively. The peaks observed at $3433 \mathrm{~cm}^{-1}$ and $3175 \mathrm{~cm}^{-1}$ were assigned to amide group $\mathrm{N}-\mathrm{H}$ asymmetric and symmetric stretching vibrations.

The $\mathrm{NH}_{2}$ deformation vibration in the FT-IR spectrum presented a very strong peak at $1614 \mathrm{~cm}^{-1}$ and was assigned to $\mathrm{NH}_{2}$ scissoring, and the theoretically scaled down value being $1613 \mathrm{~cm}^{-1}$ which was well within the region of $1650-1590 \mathrm{~cm}^{-1}$ as reported in literature. ${ }^{29}$ The scaled down frequency at $1069 \mathrm{~cm}^{-1}$ may be assigned to $\mathrm{NH}_{2}$ rocking vibration and in the recorded FT-IR spectrum, it was observed at $1080 \mathrm{~cm}^{-1}$. The peak at $579 \mathrm{~cm}^{-1}$ in FT-Raman could be attributed to $\mathrm{NH}_{2}$ wagging mode and it was correlated with the computed value of $577 \mathrm{~cm}^{-1}$.

The carbonyl stretching vibration of $\mathrm{C}=\mathrm{O}$ group is expected to appear at the region of $1680-1630 \mathrm{~cm}^{-1}$ for amides. The very strong $\mathrm{C}=\mathrm{O}$ band observed at $1735 \mathrm{~cm}^{-1}$ corresponds to the stretching vibration of $\mathrm{C}=\mathrm{O}$ group of $3 \mathrm{~A} 4 \mathrm{MBA}$. In-plane deformation of $\mathrm{C}=\mathrm{O}$ possibly occurs in the region of $750-695 \mathrm{~cm}^{-1}$ and it is observed as a medium intensity band at $726 \mathrm{~cm}^{-1}$ in FT-Raman. The calculated vibrations for these bands are in excellent consistency with the experimentally obtained results.

Band mixing leads to difficulty in identifying the $\mathrm{C}-\mathrm{N}$ vibrations. However, with the help of theoretical calculations and relative percentage of weight stretching and deformation, vibrations of $\mathrm{C}-\mathrm{N}$ are identified and assigned in this study. The vibrational assignments for amides appeared in the region 1420 $1450 \mathrm{~cm}^{-1} .^{28}$ In 3A4MBA, intense C-N stretching bands are found at $1457,1287 \mathrm{~cm}^{-1}$ in FT-IR spectrum. DFT at B3LYP/6-311++G** level computed frequencies showed an excellent agreement with the corresponding experimental ones. Amino group $\mathrm{C}-\mathrm{N}$ stretching vibration got slightly lowered due to the formation of intramolecular hydrogen bonding.

3.8c Methoxy group vibrations: Basically, nine fundamentals are assigned to the $\mathrm{CH}_{3}$ group, namely, symmetric stretch; in-plane stretch (i.e., in-plane hydrogen deformation modes), symmetric bending, in-plane rocking, out-of-plane rocking, twisting hydrogen bending modes and out-of-plane hydrogen bending modes inclusive of $\mathrm{CH}_{3}$ out-of-plane stretch. $\mathrm{CH}_{3}$ out-of-plane bending modes of the $\mathrm{CH}_{3}$ group are also found. $\mathrm{CH}_{3}$ stretching modes appear at 2959,2919, $2841 \mathrm{~cm}^{-1}$ in the FT-Raman spectrum of 3A4MBA. Symmetric 
bending vibration was observed at $1343 \mathrm{~cm}^{-1}$ in FTRaman, in-plane and out-of-plane bending vibrations are at $1448,1380 \mathrm{~cm}^{-1}$, respectively. Torsion vibration occurred at $249 \mathrm{~cm}^{-1}$ in the FT-Raman spectrum. Inplane bending vibration of methoxy group appeared at 472 and $384 \mathrm{~cm}^{-1}$ in the FT-Raman spectrum and these appear to have good agreement with the calculated spectrum.

\subsection{Low frequency hydrogen bonding vibrations}

The interaction between the hydrogen donor and acceptor groups leads to the formation of new vibrational degrees of freedom which appear at lower frequencies in the range between 50 and $300 \mathrm{~cm}^{-1}{ }^{30}$ These modes arise due to the lattice vibrations and are referred to as external modes. Rotational and translational vibrations along with the vibrations of hydrogen modes are usually involved in external modes. The modes of hydrogen bond are found to be weak in the FT-Raman spectrum with overlapping of liberation modes. The peak in the FT-Raman at $72 \mathrm{~cm}^{-1}$ is assigned to the translational motion of the hydrogen bonded molecules while the intense band at $107 \mathrm{~cm}^{-1}$ is assigned to the rotational motion. In general, these lattice modes are found to be stronger in intensity than other higher frequency bands.

\subsection{Topological study (AIM)}

'Atoms in molecules' (AIM) is an attractive theory for the illustration of the molecular topology as it determines the Bond Critical Points (BCP) between neighboring atoms. ${ }^{31,32}$ Localizing the bond critical points, several properties can be calculated at their position in space. Amongst these, the most important one is $\rho \mathrm{BCP}$ or the charge density at the bond critical point which has a minimal value along the line of the bond. The next useful quantity to characterize a bond is the Hessian $(3 \times 3)$ matrix of second order partial derivatives with respect to coordinates of the charge density. The coordinate invariant (ordered) eigenvalues $\lambda 1<\lambda 2<\lambda 3$ arise from the diagonalization of this matrix. Using the Eigenvalues, the Laplacian of the charge density $\left(\nabla^{2} \rho_{\mathrm{BCP}}\right)$ at the bond critical point can be calculated. According to Koch and Popelier criteria, ${ }^{33}$ for closedshell interactions found in ionic bonds, hydrogen bonds, and van der Waals molecules, the $\nabla 2 \rho_{\mathrm{BCP}}$ should be positive (in the range $0.015-0.15$ a.u.) and low value of $\rho$ (0.002-0.040 a.u.). For electron density analysis of atoms, the positions of $(3,-3)$ critical points are nearly identical to nuclear positions, hence, $(3,-3)$ is called nuclear critical point (NCP) which usually occurs in heavy atoms whereas $(3,-1)$ is called the bond critical point $(\mathrm{BCP}),(3,+1)$ and $(3,+3)$ are ring critical point $(\mathrm{RCP})$ and cage critical point $(\mathrm{CCP})$, respectively. The molecular graph of the 3A4MBA using Multiwfn program $^{34}$ shown in Figure 9 gives BCPs, RCPs and NCPs (topological parameters) along with the bond paths.

The energy of the N-H- - -O hydrogen bond has been calculated by the relationship $\mathrm{E}_{\mathrm{HB}}=\mathrm{V}\left(\mathrm{r}_{\mathrm{BCP}}\right) / 2$ described by Espinosa et al. ${ }^{35}$ Intramolecular hydrogen bonding energy of $3 \mathrm{~A} 4 \mathrm{MBA}$ has been calculated to be $-20.053 \mathrm{kcal} / \mathrm{mol}$. Hydrogen bond length is $2.431 \AA$ which is less than van der Waals radius $(2.7 \AA)$. Figure 9 shows one more BCP between $\mathrm{H} 11$ and $\mathrm{H} 12$ which in turn gives one more ring critical point also. This $\mathrm{H}-\mathrm{H}$ interaction arises because of the inter atomic distance $(2.02 \mathrm{~A})$ is less than the sum of their van der Waals radii $(2.4 \AA)$. This results in an increase in the repulsive contributions of their energies and this change is dominated by an increase in the magnitude of the attractive interaction of the protons with the electron density distribution. ${ }^{36}$

Johnson and co-workers ${ }^{37}$ developed an approach to investigate the weak interactions in real space based on the electron density and its derivatives. The reduced density gradient (RDG) is a fundamental dimensionless quantity coming from the density and its first derivative:

$$
R D G(r)=\frac{1}{2\left(3 \pi^{2}\right)^{1 / 3}} \frac{|\nabla \rho(r)|}{\rho(r)^{4 / 3}}
$$

The weak interactions can be isolated as regions with low electron density and low RDG value. The density values of the low-gradient spikes (the plot of RDG versus $\rho$ ) appear to be an indicator of the interaction strength. The sign of $\lambda 2$ is utilized to distinguish the bonded $(\lambda 2<0)$ from nonbonded $(\lambda 2>0)$ interactions.

RDG is a way to distinguish weak interaction region from other regions. RDG provides a rapid and rich representation of van der Waals interactions, hydrogen bonds and steric clashes. The three-dimensional molecular structure defines covalent bonds, however, the noncovalent interactions are hidden within the voids in the bonding network. RDG is one of the tools to view and analyze the covalent and electrostatic interactions. ${ }^{37}$ The plot of the RDG versus the electron density, $\rho$ multiplied by the sign of $\lambda 2$ can allow analysis and visualization of a wide range of interaction types. One or more spikes are found in the low-density, low-gradient region which is indicative of weak noncovalent interactions. In the present case, as seen in Figure 10, such a weak noncovalent interaction between O16- - - H15-N13 is observed. Liberation modes higher negative values of 
<smiles>CCCOC1OB(O)OC(OC(=O)O)OC1OC(=O)O</smiles>
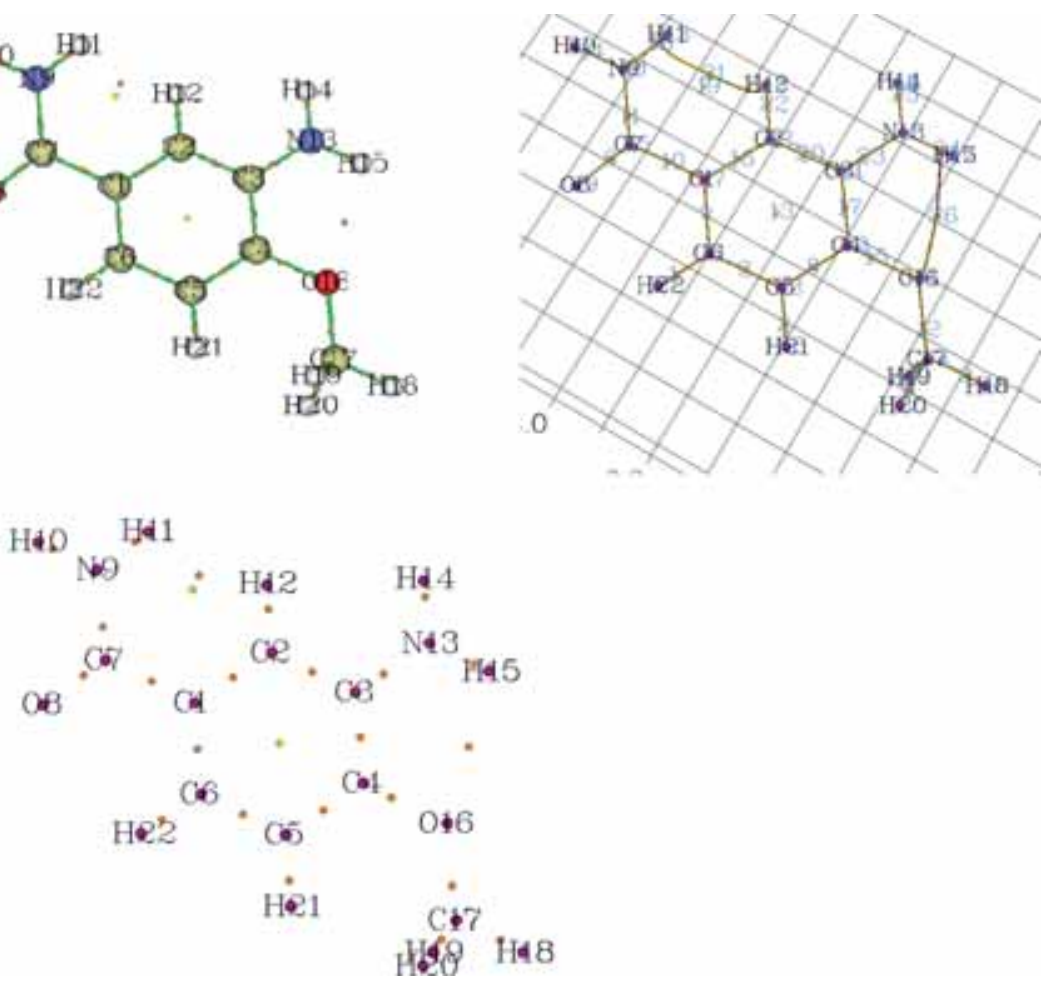

Figure 9. Molecular graph of 3A4MBA. Bond critical points (small orange spheres), ring critical points (small yellow spheres), nuclear critical point (small magenta spheres) and bond paths (brown lines) using Multiwfn program.

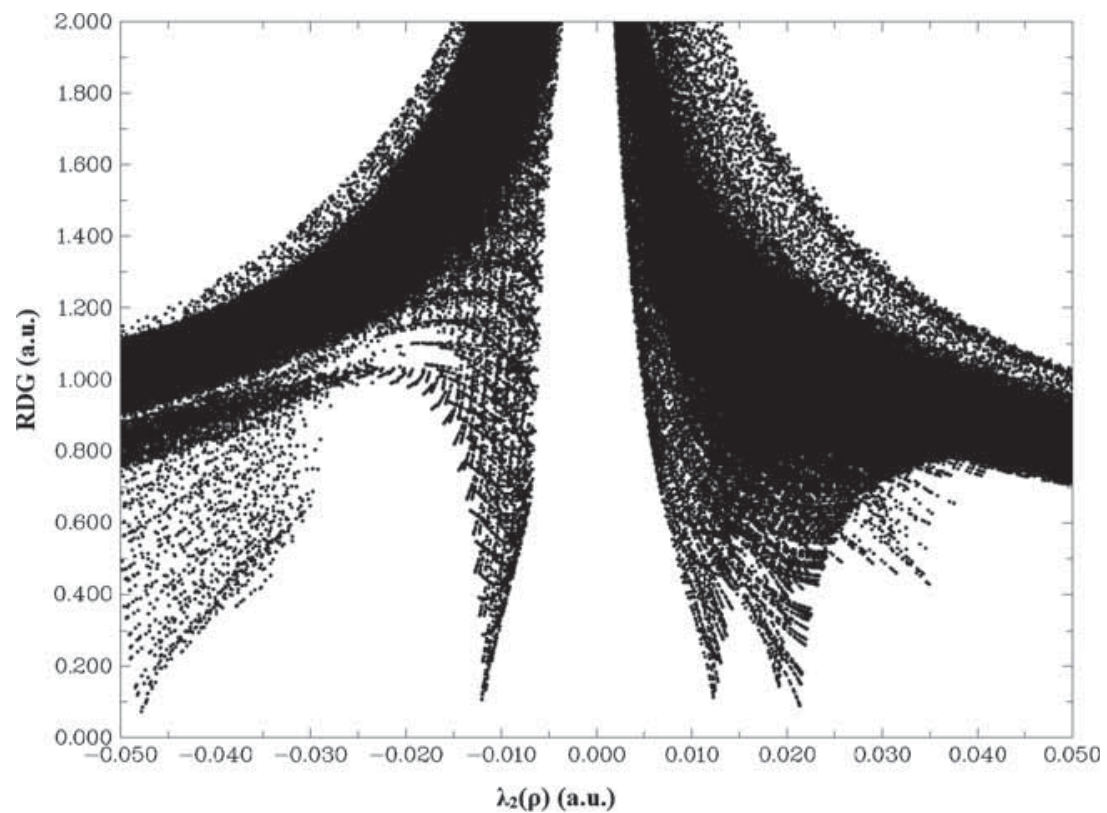

Figure 10. Plots of the RDG $v s(\lambda 2) \rho$, the electron density q multiplied by k2 for 3A4MBA.

( $\lambda 2) \rho$ are indicative of stronger attractive interactions, while large and positive values indicate repulsive interaction. Spikes found in the large positive region show
H11-H12 type of non-bonded repulsive interaction while values near zero indicate very weak, van der Waals interactions. 


\section{Conclusions}

Intramolecular hydrogen bonding with a distance of $2.431 \AA$ between the $\mathrm{O} 16$ and $\mathrm{H} 15$ atoms was confirmed in the title molecule. The stabilization energy and the values of donor and acceptor occupancies resulting from the lone pair transition $\mathrm{LP}(\mathrm{O} 16) \rightarrow \sigma^{*}$ (N13-H15) confirmed very well the weak intramolecular hydrogen bonding from NBO analysis. Further, the effective $\pi$-electron delocalization was confirmed through the transitions in the ring. Evidence was found for the existence of weak intramolecular hydrogen bonding from MEP and natural charge analysis. The lesser impact of hydrogen bonding in the ring system was indicated by HOMA index. Topological analysis was used to calculate the intramolecular hydrogen bonding energy of 3A4MBA which was found to be $-20.053 \mathrm{kcal} / \mathrm{mol}$. The theoretically predicted vibrational modes are in good agreement with the experimental ones. The incidence of low frequency bands at 72 and $107 \mathrm{~cm}^{-1}$ in the experimental FT-Raman spectrum further confirmed the intramolecular hydrogen bonding.

\section{Supplementary Information (SI)}

All additional information pertaining to geometrical parameters of 3-amino-4-methoxy benzamide are given in Table S1 and Natural charge in Table S2. Information regarding vibrational studies are given in Tables S3, S4 and S5. Supplementary Information is available at www.ias.ac.in/chemsci.

\section{Acknowledgements}

The authors are thankful to Sophisticated Analytical Instrumentation Facility (SAIF), IIT Madras for FT-Raman studies. The authors are indebted to the management of Sri Paramakalyani College for the facilities provided through the research centre.

\section{References}

1. Clark J 2004 http://www.chemguide.co.uk/organicprops/ amides/background.html (accessed on 28 February 2016)

2. http://en.wikipedia.org/wiki/Benzamide (accessed on 10 October 2016)

3. Powers J P, Yosup Rew, Xuelei Yan 2010 Benzamide derivatives and uses related thereto US Patent 7659287 B2

4. Gawronski J, Kołbon H and Kwit M 2002 J. Stereo Chem. 785

5. Dewis M L, Merrit D, Miller K, Chen Z and Reiber L 2009 Benzamide Compounds Useful as High Potency Sweet Taste Enhancers US Patent 20090047379
6. Kawski P, Kochel A, Perevozkina M G and Filarowski A 2006 The intramolecular hydrogen bond in 2-hydroxybenzamides J. Mol. Struct. 79065

7. Arslan H, Florke U, Kulcu N and Binzet G 2007 The molecular structure and vibrational spectra of 2chloro-N-(diethylcarbamothioyl)benzamide by HartreeFock and density functional methods Spectrochim. Acta A 681347

8. Arjunan V and Mythili C V 2011 Experimental and theoretical investigations of benzamide oxime Spectrochim. Acta A 79245

9. Muthu S, Rajamani T, Karabacak $M$ and Asiri A M 2014 Vibrational and UV spectra, first order hyperpolarizability, NBO and HOMO-LUMO analysis of 4-chloro-N-(2-methyl-2,3-dihydroindol-1-yl)-3sulfamoyl-benzamide Spectrochim. Acta A 1221

10. Jezierska A, Panek J J and Mazzarello R 2009 Structural and electronic structure differences due to the $\mathrm{O}-\mathrm{H}$. . O and $\mathrm{O}-\mathrm{H}$. . S bond formation in selected benzamide derivatives: A first-principles molecular dynamics study Theor. Chem. Acc. 124319

11. Ushakumari L, Varghese H T, Panicker C Y, Ertan T and Yildiz I 2008 Vibrational spectroscopic studies and DFT calculations of 4-fluoro- $N$-(2-hydroxy-4nitrophenyl)benzamide J. Raman Spectrosc. 391832

12. Arjunan V, Kalaivani M, Ravindran $\mathrm{P}$ and Mohan S 2011 Structural, vibrational and quantum chemical investigations on 5-chloro-2-hydroxybenzamide and 5-chloro-2-hydroxybenzoic acid Spectrochim. Acta A 791886

13. Frisch M J et al. 2004 Gaussian, Inc., Wallingford CT

14. Becke A D 1993 Density-functional thermochemistry. III. The role of exact exchange J. Chem. Phys. 985648

15. Becke A D 1998 Density-functional exchange-energy approximation with correct asymptotic behaviour Phys. Rev. A $\mathbf{3 8} 3098$

16. Pulay P, Fogarasi G, Pang F and Boggs J E 1979 Systematic ab initio gradient calculation of molecular geometries, force constants, and dipole moment derivatives J. Am. Chem. Soc. 1012550

17. Arjunan V, Suja Ravi Isaac A, Rani T, Mythili C V and Mohan S 2011 Density functional theory studies on vibrational and electronic spectra of 2-chloro-6methoxypyridine Spectrochim. Acta A 781625

18. James C, Amal Raj A, Reghunathan R, Hubert Joe I and Jayakumar V S 2006 Structural conformation and vibrational spectroscopic studies of 2,6-bis $(p-N, N$-dimethyl benzylidene)cyclohexanone using density functional theory J. Raman Spectrosc. 371381

19. Scrocco E and Tomasi J 1978 Electronic Molecular Structure, Reactivity and Intermolecular Forces: An Euristic Interpretation by Means of Electrostatic Molecular Potentials Adv. Quantum Chem. 11115

20. Murray J S and Sen K 1996 In Molecular Electrostatic, Potentials Concepts and Applications (Amsterdam: Elsevier)

21. Sobczyk L, Grabowski S J and Krygowski T M 2005 Interrelation between $\mathrm{H}$-bond and Pi-electron delocalization Chem. Rev. 1053513

22. Sundius $\mathrm{T} 2002$ Scaling of ab initio force fields by MOLVIB Vib. Spectrosc. 2989

23. Sundius T 2002 MOLVIB (v.7.0); Calculation of Harmonic Force Fields and Vibrational Modes of Molecules, QCPE, Program No. 807 (2002) 
24. Sundius T 1990 MOLVIB: A program for harmonic force field calculations, CPE Program No. $604 \mathrm{~J}$. Mol. Struct. 218321

25. Fogarasi G, Pulay P and Durig J R 1985 In Vibrational Spectra and Structure 4 (Amsterdam: Elsevier) Ch. 3 p. 125

26. Fogarasi G, Zhov X, Taylor P W and Pulay P 1992 The calculation of $\mathrm{ab}$ initio molecular geometries: Efficient optimization by natural internal coordinates and empirical correction by offset forces J. Am. Chem. Soc. 1148191

27. Varsanyi G 1969 In Vibrational spectra of Benzene Derivatives (New York: Academic press)

28. Socrates G 2001 In Infrared and Raman Characteristic Group Frequencies-Tables and Charts 3rd ed. (Chichester: John Wiley)

29. Tellez C A, Hollauer E, Felcman J, Lopes D C N and Cattapan R A 2002 Fourier-transform infrared and Raman spectra, and ab initio calculations for cadmium-n-di-isopropylphosphorylguanidine-di-chloride (CdDPGCl2) complex Spectochim. Acta A $\mathbf{5 8} 1853$

30. Padmaja L, Vijayakumar T, Hubert Joe I, Reghunadhan Nair C P and Jayakumar V S 2006 Vibrational spectral studies and the non-linear optical properties of a novel
NLO material L-prolinium tartrate J. Raman Spectrosc. 371427

31. Bader R F W 1990 In Atoms in Molecules: A Quantum Theory (Oxford: Oxford University Press)

32. Bader R F W 1991 A quantum theory of molecular structure and its applications Chem. Rev. 91893

33. Koch U and Popelier P L A 1995 Characterization of $\mathrm{C}-\mathrm{H}-\mathrm{O}$ Hydrogen Bonds on the Basis of the Charge Density J. Phys. Chem. 999747

34. Lu T and Chen F 2012 Multiwfn: A multifunctional wavefunction analyzer J. Comput. Chem. 33580

35. Espinosa E, Molins E and Lecomte C 1998 Hydrogen bond strengths revealed by topological analyses of experimentally observed electron densities Chem. Phys. Lett. 285170

36. Matta C F, Hernandez-Trujillo J, Tang T-H and Bader R F W 2003 Hydrogen- Hydrogen Bonding: A Stabilizing Interaction in Molecules and Crystals Chem. Eur. J. 91940

37. Johnson E R, Keinan S, Mori-Sánchez P, ContrerasGarcía J, Cohen A J and Yang W 2010 Revealing noncovalent interactions J. Am. Chem. Soc. 1326498 